Ann. sci. forest., 1977, 34 (2), 89-109.

\title{
Influence de la fertilisation minérale sur la fructification du Hêtre (Fagus silvatica)
}

\author{
F. LE TACON * et H. OSWALD ** \\ avec la collaboration de \\ Maryse BITSCH *, D. BOUCHARD *, A. DIVOUX ** \\ * Station de Recherches sur les Sols forestiers et la Fertilisation, \\ ** Station de Sylviculture ef de Production. \\ Centre National de Recherches forestières, I.N.R.A. \\ Champenoux, 54280 Seichamps
}

\begin{abstract}
Résumé
Un dispositif installé dans l'Ouest de la France (Fougères) et un autre installé dans l'Est (Darney) montrent que la fertilisation a un effet considérable sur la fructification du Hêtre. A Fougères, la fertilisation permet d'augmenter le nombre de fleurs mâles de 70 p. 100 et surtout de multiplier le nombre de fleurs femelles par 2,7 .

La fertilisation agit également en augmentant le nombre de faînes viables par rapport au nombre de faînes avortées.

Ces différents effets de la fertilisation se conjuguent et permettent de multiplier le nombre de faînes viables par 3,4 à Fougères et par 3,38 à Darney.

L'effet de la fertilisation semble surtout dû à l'azote et, à degré moindre, au phosphore. Une fertilisation de type NP semble donc à conseiller.

La fertilisation a un effet sur la fructification du Hêtre, même sur des peuplements de productivité élevée comme à Darney et même sur des peuplements âgés (Fougères : 140 ans). L'effet de la fertilisation semble indépendant du type d'humus, au moins dans une certaine gamme puisqu'il est aussi important sur mor, sur moder que sur mull acide.

Enfin, à Darney, la fertilisation est encore efficace trois ans après l'apport d'engrais.

Plusieurs problèmes restent encore à résoudre :

- temps d'action d'un seul apport fertilisant ;

- effet sur des stations à mull mésotrophe, mull eutrophe et mull calcique.
\end{abstract}

\section{Introduction}

La régénération naturelle du Hêtre se heurte à de nombreuses difficultés, dont les premières sont l'espacement des faînées (souvent 5 à 10 ans) et leur insuffisance.

L'espacement des faînées semble êtrc d'origine climatique, ce qui élimine presque toutes les possibilités d'intervention. L'abondance d'une fructification par contre, si elle est encore sous la dépendance de fccteurs climatiques, semble aussi dépendre de facteurs édaphiques. 
Parmi ces facteurs, la nutrition minérale semble avoir un rôle certain si nous nous référons aux quelques rares travaux qui ont été effectués dans ce domaine.

C'est ainsi que Borchers, Gussone et Kramer, en République Fédérale d'Allemagne, ont montré en 1964 qu'une fertilisation, et en particulier une fertilisation azotée, pouvait accroître la faînée. Toujours en Allemagne, Hausser en 1971, a obtenu une multiplication du nombre de faînes par 4 environ et une augmentation du poids moyen des faînées de l'ordre de 20 p. 100 avec une fertilisation complète.

De même Weissen, en Belgique, a obtenu une augmentation de 50 p. 100 de la faînée dans un dispositif installé à Bertrix en 1967 par un apport d'azote (150 kilogrammes de $\mathrm{N}$ /hectare sous forme d'urée) (communication personnelle).

Cet effet de la fertilisation avait été observé uniquement en année de faînée. D'autre part, la fertilisation n'avait eu aucun effet, ni sur le pouvoir germinatif des faînes, ni sur la croissance des semis.

En France, sur le Chêne, Garbaye et Leroy (1974) ont obtenu à Bercé une augmentation de 60 p. 100 du nombre de glands par fertilisation, mais aucune amélioration de la qualité de la glandée (poids des glands, pouvoir germinatif).

Ces différents résultats nous ont amené à installer un essai de fertilisation dans un peuplement âgé de Hêtre en forêt domaniale de Fougères (Ille-et-Vilaine).

Nous avons choisi cette forêt d'une part en raison de la pauvreté relative des sols et d'autre part, en raison de la fréquence élevée des faînées. Le climat océanique à hiver doux et la rareté des gelées tardives semblent en effet favoriser la fréquence des faînées.

Les résultats obtenus dans le dispositif de Fougères spécialement conçu pour l'étude de la fructification du hêtre nous ont incité à exploiter un autre dispositif expérimental en forêt domaniale de Darney (Vosges), installé sur des sols beaucoup plus riches, et dont le but initial était de déterminer l'influence de la fertilisation sur la production et la qualité du bois de hêtre.

\section{2. - Les conditions de milieu}

\section{En forêt de Fougères}

La forêt de Fougères est située à une trentaine de kilomètres de la baie du MontSt-Michel entre 115 et 190 mètres d'altitude. La pluviosité est de 880 millimètres, régulièrement répartie toute l'année avec un léger maximum en octobre, novembre et décembre. La température moyenne de juillet est de $17,3^{\circ}$ et celle de janvier de 3,90.

L'E.T.P. calculée suivant la méthode de Thornthwaite est de 645 millimètres. Le nombre de jours de gelée dans l'année est en moyenne de 35.

La forêt de Fougères repose presque entièrement sur du granite, dit granite de Vire. Ce granite est partout recouvert par un limon éolien d'épaisseur moyenne comprise entre 1 et 2 mètres (Toutain, 1966).

Le sol dans le dispositif expérimental, parfaitement représentatif de l'ensemble de la forêt, est un sol brun marmorisé à moder-mor, avec micropodzolisation secondaire de surface.

Il existe un $A_{0}$ de 4 centimètres d'épaisseur avec des couches $L, F$ et $\mathrm{H}$ bien individualisées. L'horizon $A_{2}$ de micropodzolisation varie de 1 à 5 centimètres ef l'horizon 
$B_{h} / B_{f e}$ de 3 à 8 centimètres. C'est un sol acide $\left(\mathrm{pH} 3,5\right.$ en $A_{0}$ et $A_{1}-4,0$ en (B)) pauvre en bases échangeables, à taux de saturation faible inférieur à 10 p. 100 dans la partie supérieure du profil, et à $\mathrm{C} / \mathrm{N}$ supérieur à 20 (27 en $\mathrm{A}_{1}$ ).

Les réserves en eau utile sont par contre élevées en raison de la texture limoneuse et de l'épaisseur des limons.

La végétation est acidiphile à base d'llex aquifolium et Rhamnus frangula dans la strate arbustive et dans la strate herbacée on trouve Deschampsia flexuosa, Vaccinium myrtillus, Molinia coerulea et Hypnum triquetrum.

\section{En forêt de Darney}

La forêt de Darney est située dans les Basses-Vosges, entre 300 et 400 mètres d'altitude. La pluviosité est de 950 millimètres, assez régulièrement répartie avec un léger maximum en juillet-août et un léger minimum en janvier-février-mars. La température moyenne de juillet est de $18^{\circ}$ et celle de janvier de $-1,7^{\circ}$.

La forêt de Darney s'étend sur le trias, du grès vosgien au Muschelkalk moyen. La hêtraie correspond essentiellement aux affleurements du grès à voltzia et du grès intermédiaire.

Sur grès à voltzia, les sols sont le plus souvent des sols bruns acides à mull acide, plus rarement à mull-moder, soit des sols bruns ocreux à moder.

Les sols bruns acides à mull acide sont caractéristiques de la hêtraie à luzulf blanche (Luzula albida).

Les sols bruns acides à mull-moder et bruns ocreux à moder sont caractéristiques de la hêtraie à canche flexueuse (Deschampsia flexuosa).

Ces deux types de hêtraie sont parmi les plus répandus dans les Basses-Vosges. Dans le dispositif de fertilisation, ces deux types de hêtraie sont représentés (tabl. 1 et 2 ).

Les réserves en eau utile de ces deux profils sont assez importantes en raison de la charge non négligeable en limons et argile et en raison de l'épaisseur du profil (1 mètre au moins).

\section{TABLEAU 1}

Hêtraie à luzule, sol brun acide à mull acide sur grès à voltzia avec légère " pollution » limoneuse

(Forêt domaniale de Darney)

Soil analysis of a beech site with Luzula albida at Darney ; acid brown soil, with acid mull on sandstone, slightly covered with eolian loam

\begin{tabular}{|c|c|c|c|c|c|c|c|c|c|c|c|}
\hline & \multirow{2}{*}{$\begin{array}{l}\text { m. o. } \\
\text { p. } 100\end{array}$} & \multirow{2}{*}{$\begin{array}{c}\text { C. } 100 \\
\text { p. }\end{array}$} & \multirow{2}{*}{$\begin{array}{l}N \\
\text { p. } 100\end{array}$} & \multirow[t]{2}{*}{$\mathrm{C} / \mathrm{N}$} & \multirow[t]{2}{*}{$\mathrm{pH}$} & \multicolumn{4}{|c|}{ m. e. $100 \mathrm{~g}$} & \multirow[t]{2}{*}{$S / T$} & \multirow{2}{*}{$\begin{array}{r}\mathrm{P}_{2} \mathrm{O}_{5} \\
\text { p. } 100\end{array}$} \\
\hline & & & & & & $\mathrm{Ca}$ & K & $\mathrm{Mg}$ & $\mathrm{T}$ & & \\
\hline$A_{1} 0-5 \ldots \ldots$ & 8,1 & 4,7 & 0,25 & 19,0 & 4,2 & 1,33 & 0,95 & 0,24 & 16,9 & 17,5 & 0,06 \\
\hline B $20-30 \ldots \ldots$ & 1,1 & 0,65 & 0,06 & 11,0 & 4,2 & 0,35 & 0,15 & 0,15 & 6,2 & 12,0 & 0,08 \\
\hline (B) $50-60 \ldots \ldots$ & & & & & 4,2 & 0,23 & 0,15 & 0,05 & 5,6 & 9,5 & 0,08 \\
\hline (B)/C $90-100 .$. & & & & & 4,3 & 0,30 & 0,30 & 0,08 & 7,4 & 7,1 & 0,07 \\
\hline
\end{tabular}

Texture sablo-limoneuse. Taux de saturation inférieur à 20 p. 100 en $A_{1}$ et à 10 p. 100 à partir de $50 \mathrm{~cm}$. C/N inférieur à 20. Teneur en phosphore moyenne à faible. 
TABLEAU 2

Hêtraie à canche flexueuse. Sol brun ocreux à moder sur grès (transition grès à voltzia grès intermédiaire) (Forêt domaniale de Darney)

Soil analysis of a beech site with Deschampsia flexuosa at Darney ; acid ochreous brown soil with moder on sandstone

\begin{tabular}{|c|c|c|c|c|c|c|c|c|c|c|c|}
\hline & \multirow{2}{*}{$\begin{array}{l}\text { m. o. } \\
\text { p. } 100\end{array}$} & \multirow{2}{*}{ p. 100} & \multirow{2}{*}{$\begin{array}{c}N \\
\text { p. } 100\end{array}$} & \multirow[t]{2}{*}{$C / N$} & \multirow[t]{2}{*}{$\mathrm{pH}$} & \multicolumn{4}{|c|}{ m. e. $100 \mathrm{~g}$} & \multirow[t]{2}{*}{$\mathrm{S} / \mathrm{T}$} & \multirow{2}{*}{$\begin{array}{c}\mathrm{P}_{2} \mathrm{O}_{5} \\
\text { p. } 1000\end{array}$} \\
\hline & & & & & & $\mathrm{Ca}$ & $\mathrm{K}$ & $\mathrm{Mg}$ & $\mathrm{T}$ & & \\
\hline$A_{2} 2-5 \ldots \ldots$ & 22,4 & 11,4 & 0,40 & 28,0 & 3,5 & 0,40 & 0,40 & 0,38 & 23,1 & 7,5 & 0,04 \\
\hline (B) $10-20 \ldots \ldots$ & & & & & 4,1 & 0,15 & 0,19 & 0,08 & 10,2 & 4,7 & 0,04 \\
\hline (B) $40-50 \ldots \ldots$ & & & & & 4,1 & 0,15 & 0,15 & 0,06 & & & 0,04 \\
\hline (B)/C 80-90... & & & & & 4,3 & 0,30 & 0,34 & 0,10 & 8,7 & 9,1 & 0,04 \\
\hline
\end{tabular}

Texture sablo-limoneuse. Taux de saturation très faible inférieur à 10 p. 100 dans tout le profil. C/N élevé. Teneur en phosphore faible.

\section{3. - Les peuplements}

\section{En forêt domaniale de Fougères}

Le peuplement est issu d'une régénération naturelle presque pure (environ 5 p. 100 de Chêne et divers) et très homogène ; il est traité en futaie régulière. La dernière éclaircie a eu lieu en 1968 (rotation 12 ans). L'âge du peuplement est de 140 ans avec une fourchette de 120 à 140 ans.

D'après les tables de production anglaises (1971) qui, selon Le Goff (1974) peuvent être utilisées dans cette région sans trop de risques d'erreur, le peuplement se situe dans la « yield class » 6 (accroissement moyen annuel maximum de 6 mètres cube bois fort tige à l'hectare), c'est-à-dire dans une classe de fertilité légèrement en dessous de la moyenne. Toutefois le nombre de tiges est un peu plus élevé (éclaircies plus faibles).

Le tableau 3 ci-dessous compare les valeurs moyennes du peuplement avec celles de la table anglaise.

TABLEAU 3

Comparaison des principales caractéristiques du peuplement de Fougères avec la classe 6 de la table de production anglaise

Principal stand characteristics of the beech stand in Fougères

\begin{tabular}{|c|c|c|c|c|}
\hline & $\begin{array}{l}\text { Peuplement } \\
\text { en F. D. } \\
\text { de Fougères }\end{array}$ & \multicolumn{3}{|c|}{ Table anglaise « Yield class » 6} \\
\hline 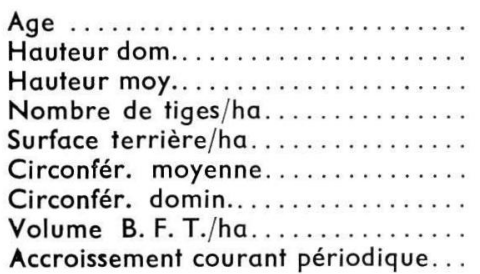 & $\begin{array}{l}130 \\
26,50 \\
25,75 \\
195 \\
32,95 \\
146 \\
167 \\
- \\
-\end{array}$ & $\begin{array}{l}120 \\
25,70 \\
- \\
174 \\
33,30 \\
155 \\
-7 \\
329 \\
4,4\end{array}$ & $\begin{array}{l}130 \\
26,10 \\
- \\
154 \\
34,20 \\
167 \\
- \\
339 \\
3,9\end{array}$ & $\begin{array}{l}140 \\
26,30 \\
137 \\
34,90 \\
179 \\
34 \overline{-} \\
3,5\end{array}$ \\
\hline
\end{tabular}


Tous les placeaux ont été inventoriés pied par pied ; la répartition des tiges par classe de circonférence est donnée dans le tableau 4 ci-dessous.

TABLEAU 4

Répartition des tiges par classe de circonférence à Fougères

Girth distribution of the stand in Fougères

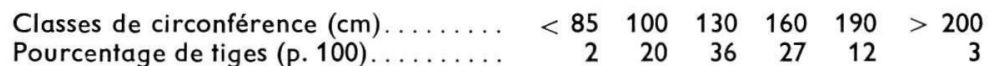

\section{En forêt domaniale de Darney}

Le peuplement est issu d'une régénération naturelle. II s'agit d'une futaie de hêtre âgée de 90 ans ( \pm 10 ans), avec un faible mélange de chêne rouvre (environ 10 p. 100). D'après la table de production - éclaircie forte - de Schober (Schober 1972) *, qui selon Le Goff (1974) peut être utilisée dans le NE de la France, le peuplement se situe en moyenne dans la première classe de fertilité.

La hauteur dominante est en moyenne de 29,8 mètres ; elle varie cependant de 25,0 mètres à 32,9 mètres. Cette variation est due à des différences d'âge pratiquement incontrôlables, et aux conditions de station (types de sol et d'humus, épaisseur des limons, etc...) qui ne sont pas homogènes sur l'ensemble du dispositif.

Ainsi, un certain nombre de placeaux correspond à la $2^{e}$ classe de fertilité de la table de production. Le tableau 5 donne les principales caractéristiques du peuplement de l'essai de Darney et les compare aux classes 1 et 2 de la table de production de Schober.

TABLEAU 5

Comparaison des principales caractéristiques du peuplement de Darney avec les classes 1 et 2 de la table de production de Schober (éclaircie forte)

Principal stand characteristics of the beech stand in Darney

\begin{tabular}{|c|c|c|c|c|c|c|c|c|c|}
\hline & \multicolumn{3}{|c|}{$\begin{array}{l}\text { Peuplement } \\
\text { de Darney }\end{array}$} & \multicolumn{6}{|c|}{$\begin{array}{l}\text { Table de production } \\
\text { Eclaircie forte (Schober) }\end{array}$} \\
\hline & \multirow{2}{*}{$\begin{array}{l}\text { Min. } \\
-\end{array}$} & \multirow{2}{*}{$\begin{array}{c}\text { Moy. } \\
90\end{array}$} & \multirow{2}{*}{$\begin{array}{c}\text { Max. } \\
-\end{array}$} & \multicolumn{3}{|c|}{ Classe 1} & \multicolumn{3}{|c|}{ Classe 2} \\
\hline Age $\ldots \ldots \ldots \ldots \ldots$ & & & & 80 & 90 & 100 & 80 & 90 & 100 \\
\hline Hauteur dom........... & 25,0 & 29,8 & 32,9 & 27,6 & 30,0 & 32,1 & 23,9 & 26,1 & 28,1 \\
\hline Nbre total des tiges/ha.... & 294 & 571 & 891 & 422 & 321 & 251 & 547 & 411 & 319 \\
\hline Surface terrière totale/ha. . & 24,6 & 32,0 & 39,2 & 24,3 & 24,7 & 25,1 & 23,4 & 24,0 & 24,4 \\
\hline Circonférence moyenne... & 69,0 & 83,9 & 103,0 & 84,8 & 98,3 & 112,1 & 73,2 & 85,8 & 98,0 \\
\hline Volume B. F. sur pied/ha.. & - & - & - & 315 & 352 & 385 & 261 & 298 & 330 \\
\hline Accroissement courant/ha. & - & - & - & 11,7 & 11,4 & 10,9 & 10,2 & 9,9 & 9,5 \\
\hline Accroissement moyen/ha.. & - & - & - & 6,6 & 7,2 & 7,6 & 5,3 & 5,8 & 6,2 \\
\hline
\end{tabular}

* Cette table est reproduite dans un recueil de tables de production présenté par N. Decourt (1973). 
Dans l'ensemble ce peuplement appartient à une classe de productivité élevée et on peut tabler en fin de révolution (environ 120 ans) sur une production moyenne annuelle de l'ordre de 8 mètres cubes de bois fort par hectare.

On remarquera également que la densité et la surface terrière sont nettement plus élevées que celles de la table de Schober.

Une étude dendrométrique plus détaillée sera faite dans un proche avenir et elle donnera les premiers résultats de l'effet de la fertilisation sur la croissance en diamètre du Hêtre.

\section{4. - Les dispositifs expérimentaux}

\section{1. - Fougères}

Les conditions de sol et de végétation ainsi que le peuplement étant parfaitement homogènes, nous avons néanmoins installé un dispositif en "blocs éclatés ". 15 placeaux de 0,36 hectare ( 60 mètres $\times 60$ mètres) ont été classés d'après leur surface terrière en 5 blocs; à l'intérieur de chaque bloc, les 3 traitements ont été affectés au hasard.

\subsubsection{La fertilisation.}

Les 3 traitements suivants ont été retenus : Témoin, NP, NPKCa. Les engrais ont été appliqués en plein à l'aide d'un épandeur monté sur tracteur selon les modalités ci-dessous :

$N$ : 100 kilogrammes de $N$ sous forme d'ammonitrate en mai 1973 + 100 kilogrammes de $\mathrm{N}$ sous forme d'ammonitrate en mai 1974.

$P$ : 150 kilogrammes de $\mathrm{P}_{2} \mathrm{O}_{5}$ sous forme de supertriple en avril 1973

$\mathrm{K}$ : 150 kilogrammes de $\mathrm{K}_{2} \mathrm{O}$ sous forme de sulfate de potassium en avril 1973

$\mathrm{Ca}: \quad 1500$ kilogrammes de $\mathrm{CaO}$ sous forme de chaux vive en avril 1973.

Les quantités d'engrais sont indiquées pour 1 hectare.

\subsubsection{L'estimation de la fructification.}

L'estimation de la fructification est une opération assez délicate en raison de sa grande variabilité à l'intérieur d'un peuplement. Afin de réduire cette variabilité nous avons basé notre échantillonnage sur un certain nombre d'arbres à l'intérieur de chaque placeau en utilisant des pièges et un ramassage au sol dans des conditions bien définies.

Dans chacun des 15 placeaux, nous avons choisi 8 arbres bien conformés parmi les dominants; ces arbres sont situés à au moins 10 mètres des bordures. De plus, ils sont relativement isolés et ont de ce fait une cime assez régulière.

Sous chacun des 120 arbres, nous avons installé d'une part un piège à mi-distance entre le tronc et l'extrémité sud de la cime, et d'autre part un carré permanent de ramassage au sol (1 mètre carré) dans la direction opposée.

Les pièges sont constitués de bacs en plastique de 50 litres avec poignées (surface réceptrice de 2166 centimètres carrés) vendus dans le commerce ; ils sont installés sur 2 piquets à 1,30 mètre du sol. Le fond des bacs est percé ( 3 trous de 20 millimètres de diamètre recouverts par une grille à mailles fines) pour permettre l'écoulement des 
eaux de pluie. Un disque en grillage à maille hexagonale est placé à environ 20 centimètres du fond et empêche efficacement une consommation par les oiseaux. Ce type de piège qui est utilisé depuis 1969 à plus de 700 exemplaires s'est avéré très pratique et peu coûteux.

Les récoltes de pièges sont effectuées périodiquement depuis avril 1973 à des intervalles variables selon la chute des fleurs mâles ou des faînes ; il y a en moyenne 14 récoltes par an.

Les ramassages au sol sont effectués une seule fois dans l'année à l'automne (novembre).

\section{2. - Forêt domaniale de Darney}

Nous avons installé 34 placeaux d'une surface unitaire de 16 ares et entourés d'une zone d'isolement de 7,5 mètres de largeur. Par suite de l'hétérogénéité des sols et des peuplements, nous avons été amenés à faire une étude très détaillée afin de pouvoir établir des blocs aussi homogènes que possible. Plus de 30 variables concernant le peuplement et le milieu ont été traitées par analyses multivariables. Finalement, nous avons retenu les variables suivantes:

- surface terrière Hêtre,

- nombre de tiges par classe de circonférence (9 classes),

- circonférence dominante (Hêtre),

- notation de l'abondance ( 0 à 5 ) de la ronce, de la luzule blanchâtre et de la canche flexueuse,

- notation des types d'humus (mull, mull-moder et moder) des placeaux.

Un classement a ensuite été effectué en fonction de ces variables suivant la méthode de Van Den Driesche.

Nous avons installé ainsi 5 blocs éclatés comportant 6 traitements (4 placeaux ont été éliminés). Les blocs 1 et 5 sont sur des stations à mull, les blocs 2 et 4 sur des stations à moder et le bloc 3 sur une station à mull-moder.

\subsubsection{La fertilisation.}

Les 6 traitements choisis sont les suivants :

1. Témoin

2. $\mathrm{Ca}_{2}: 1500$ kilogrammes de $\mathrm{CaO}$ à l'hectare sous forme de calcaire

3. $\mathrm{P}: 240$ kilogrammes de $\mathrm{P}_{2} \mathrm{O}_{5}$ à I'hectare sous forme de supertriple à 45 p. 100

4. $\mathrm{PCa}_{1}: 240$ kilogrammes de $\mathrm{P}_{2} \mathrm{O}_{5}$ à l'hectare sous forme de scories à 16 p. 100 apportant en plus 675 kilogrammes de $\mathrm{CaO}$ à l'hectare.

5. $\mathrm{NPCa}_{2} \mathrm{~K}: 200$ kilogrammes d'azote sous forme d'ammonitrate à $33 \mathrm{p}$. 100

240 kilogrammes de $\mathrm{P}_{2} \mathrm{O}_{5}$ à l'hectare sous forme de scories à 16 p. 100

1500 kilogrammes de $\mathrm{CaO}$ à l'hectare dont 675 kilogrammes provenant des scories et 825 kilogrammes par apport de calcaire 150 kilogrammes de $\mathrm{K}_{2} \mathrm{O}$ sous forme de sulfate de potassium à 50 p. 100 
6. $\mathrm{NPCa}_{1} \mathrm{~K}: 200$ kilogrammes d'azote sous forme d'ammonitrate à 33 p. 100 240 kilogrammes de $\mathrm{P}_{2} \mathrm{O}_{5}$ sous forme de scories à $16 \mathrm{p}$. 100

675 kilogrammes de $\mathrm{CaO}$ apportés par les scories

150 kilogrammes de $\mathrm{K}_{2} \mathrm{O}$ sous forme de sulfate de potassium à 50 p. 100.

Le phosphore, le calcium et le potassium ont été apportés en surface au cours de l'hiver 1971-1972 ; l'azote a été apporté en 2 fois :

- 100 kilogrammes au printemps 1972

- 100 kilogrammes au printemps 1973

\subsubsection{Estimation de la fructification.}

Le dispositif n'ayant pas été prévu au départ pour l'estimation de la fructification du hêtre, les faînes et les cupules ont été ramassées au sol en janvier 1975.

Dans chacun des 30 placeaux, nous avons choisi 5 arbres dominants situés à au moins 5 mètres des bordures. Sous chacun de ces arbres, nous avons installé un carré de ramassage de 1 mètre de côté, à mi-distance entre le tronc et l'extrémité sud de la cime.

L'estimation de la faînée a donc été faite par défaut (disparition des faînes entre octobre 1974 et janvier 1975). Par contre, l'estimation de la fructification par les cupules peut être considérée comme exacte ; elle constitue un indice du potentiel de fructification.

En effet les rongeurs ne s'attaquent pratiquement pas aux cupules.

\section{3. - L'analyse des données}

Tous les calculs ont été faits à la station de Biométrie du C.N.R.F.-I.N.R.A. dans le cadre du self-service et faisaient largement appel aux procédures standardisées de la bibliothèque des programmes, notamment pour l'analyse de variance (Masson, Badia et Gigout, 1975 ; Bienaimé et Gigout, 1975).

Le modèle unique finalement retenu pour l'analyse de variance est un modèle interactif :

$$
Y_{i j k}=\mu+\alpha_{i}+\beta_{j}+(\alpha \times \beta)_{i j}+\varepsilon_{i j k}
$$

$\alpha_{i}$ : facteur bloc, niveau $5,(5) *$;

$\beta_{\mathbf{i}}$ : facteur traitement, niveaux : $3,(6)$;

$(\alpha \times \beta)_{\mathrm{ij}}$ : interaction bloc $\times$ traitement.

Les $\mathrm{k}$ arbres échantillonnés à l'intérieur d'une parcelle unitaire sont considérés comme une répétition. II y a cependant une restriction à la randomisation dans la mesure où les répétitions (arbres échantillonnés) se trouvent à l'intérieur d'une même parcelle unitaire.

L'analyse porte sur 27, (16) variables, y compris les diverses transformations (dénombrements : $\sqrt{x+1}$, pourcentages : $\arcsin \sqrt{x}$ ).

L'interprétation des résultats a été faite sur des variables transformées.

D'une manière générale, l'effet traitement est hautement significatif, mais pour certaines variables, on observe également un effet bloc et ou une interaction bloc $x$ traitement significatifs.

\footnotetext{
* Les nombres entre parenthèses concernent les niveaux dans le dispositif de Darney.
} 
Le modèle à 2 facteurs contrôlés (bloc et traitement qui se déduit d'ailleurs immédiatement du modèle interactif utilisé), ne met cependant pas en évidence un effet bloc significatif à un seuil égal ou inférieur à 5 p. 100 aussi bien à Fougères qu'à Darney.

La figure 1 représente les valeurs non transformées des placettes unitaires pour la somme des fleurs femelles et des cupules récoltées en 1974 dans les pièges du dispositif de Fougères. Les blocs 1 et 5 sont différents des blocs 2,3 et 4 ; les traitements 1 , 2 et 3 sont différents entre eux et l'interaction est essentiellement due à la valeur très élevée du traitement 3 dans le bloc 5 .

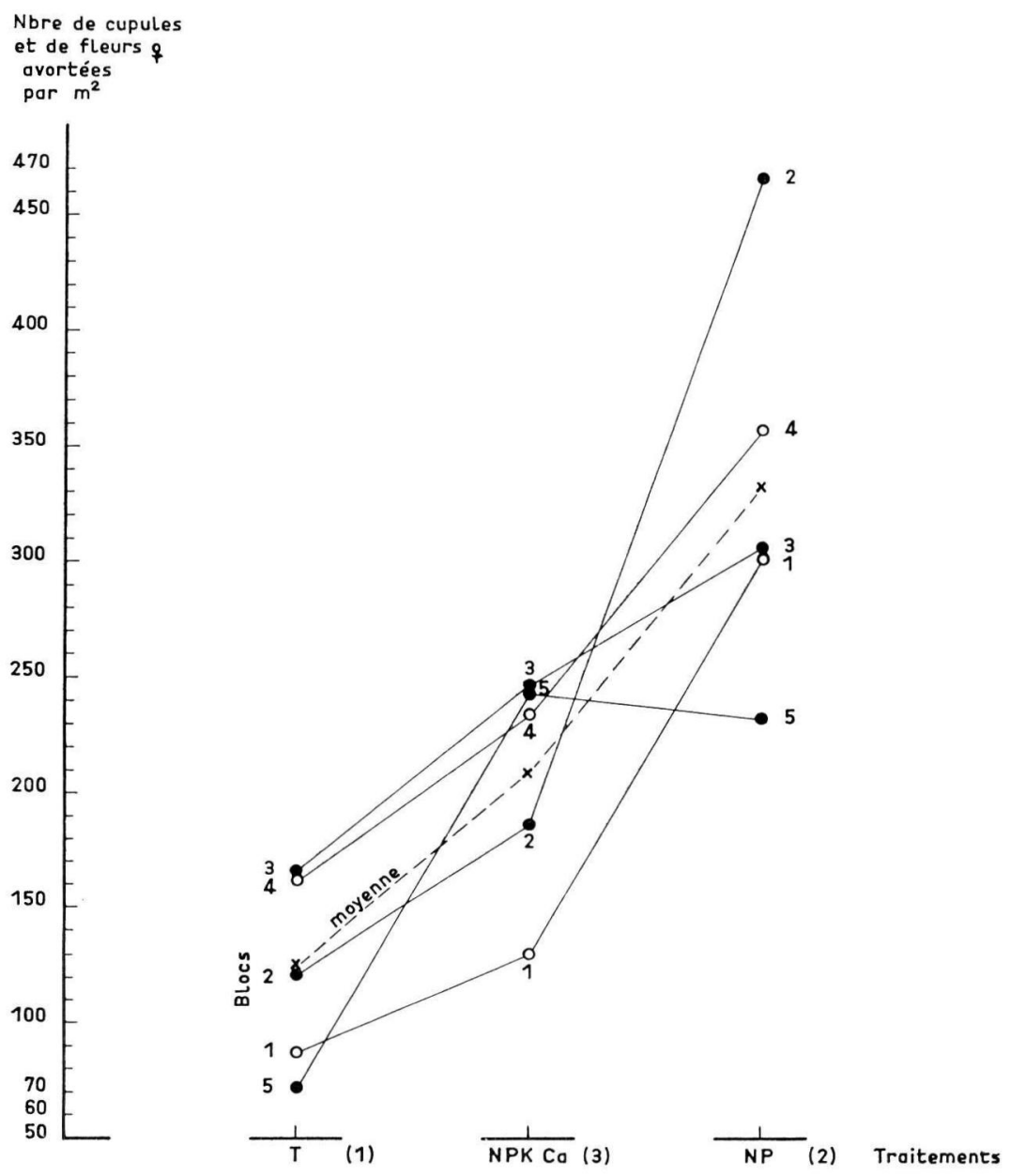

FIG. 1. - Nombre de cupules et de fleurs femelles (= estimation du nombre total des fleurs femelles à partir de pièges) en fonction des traitements en $F$. D. de Fougères ; représentation de l'interaction bloc $\times$ traitement.

Number of cupules and female flowers (= estimation of the total number of female flowers from seed traps) of the different treatments in the state forest of Fougères ; representation of the bloc $\times$ treatment interaction. 
La figure 2 montre également un effet bloc et un effet interaction bloc $x$ traitement significatifs en ce qui concerne le nombre de cupules (valeurs non transformées) dans le dispositif de Darney. On remarquera notamment le comportement du bloc 3 d mull-moder et la valeur très élevée du témoin dans le bloc 2 .

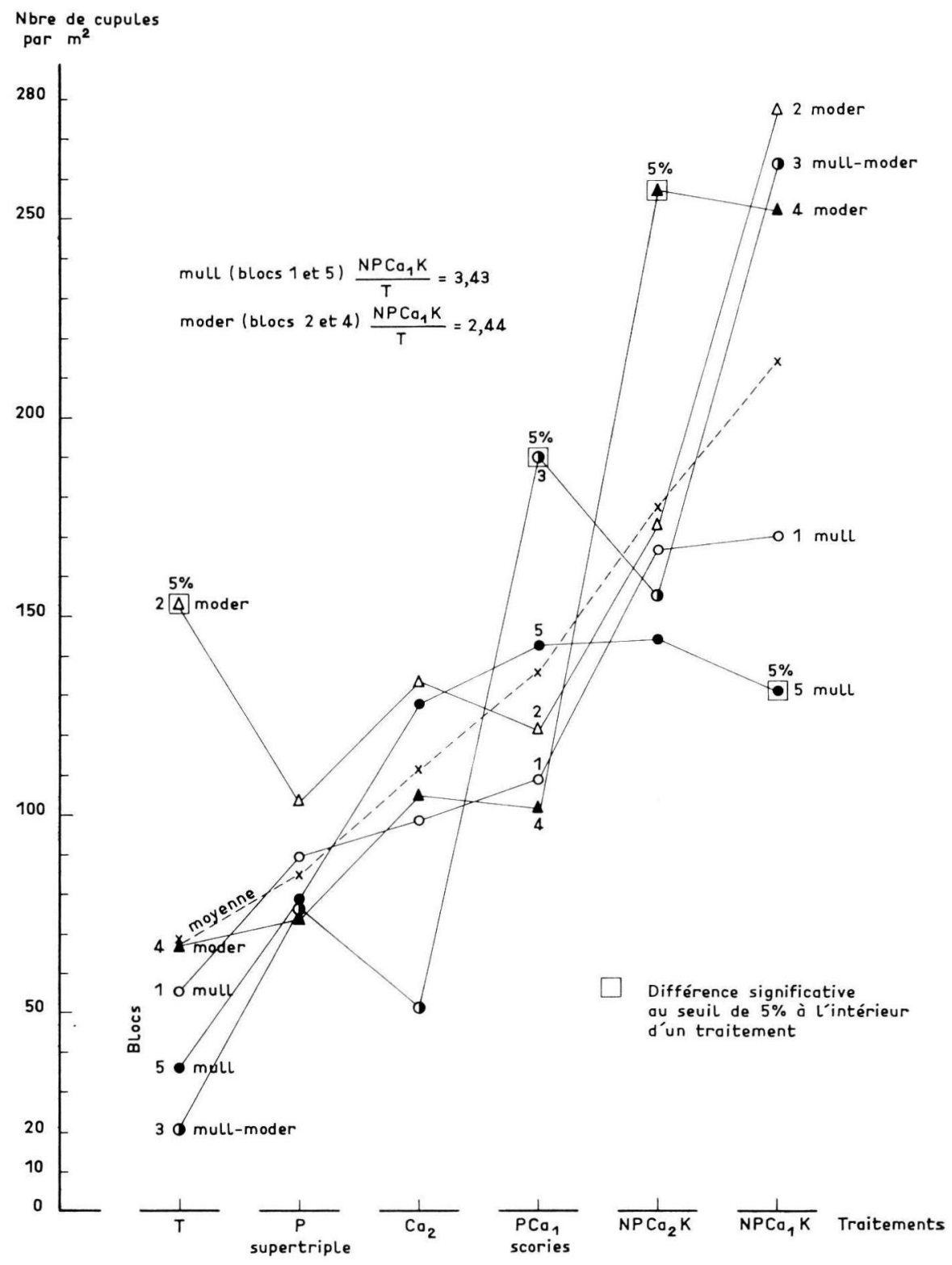

FIG. 2. - Nombre de cupules (ramassage au sol) en fonction des traitements en F.D. de Darney ; représentation de l'interaction bloc $\times$ traitement.

Number of cupules (collected on the ground) of the different treatments in the state forest of Darney : representation of the bloc $\times$ treatment interaction. 
Le modèle interactif utilisé a finalement permis de mieux cerner les effets souvent complexes de la fertilisation.

\section{5. - Résultats}

\section{1. - Forêt domaniale de Fougères}

La fructification a été nulle ou très faible en 1973 quel que soit le traitement. On trouvait en moyenne 70 fleurs mâles, 13 cupules ou fleurs femelles, 2,7 faînes viables et 4,7 faînes avortées par mètre carré. L'analyse de variance effectuée sur les données 1973 fait cependant déjà apparaître une certaine tendance d'un effet de fertilisation, mais seul le poids sec total des faînes viables du traitement NP est significativement différent des autres traitements. II n'est pas impensable que la fertilisation appliquée au printemps 1973 ait pu avoir un léger effet, non pas sur l'induction florale, mais sur le développement de la fructification de la même année.

\subsubsection{Récolte des pièges (1974).}

\subsubsection{Influence de la fertilisation sur la mise à fleur.}

Fleurs mâles (fig. 3).

Les fleurs mâles sont comptées par récolte dans les pièges. Ces fleurs sont très légères et facilement emportées par le vent. Les fleurs ramassées dans un piège ne proviennent pas forcément toutes de l'arbre sous lequel est situé le piège. De même, malgré la distance de 20 mètres séparant généralement 2 pièges de 2 placeaux différents il y a probablement eu des interférences entre traitements.

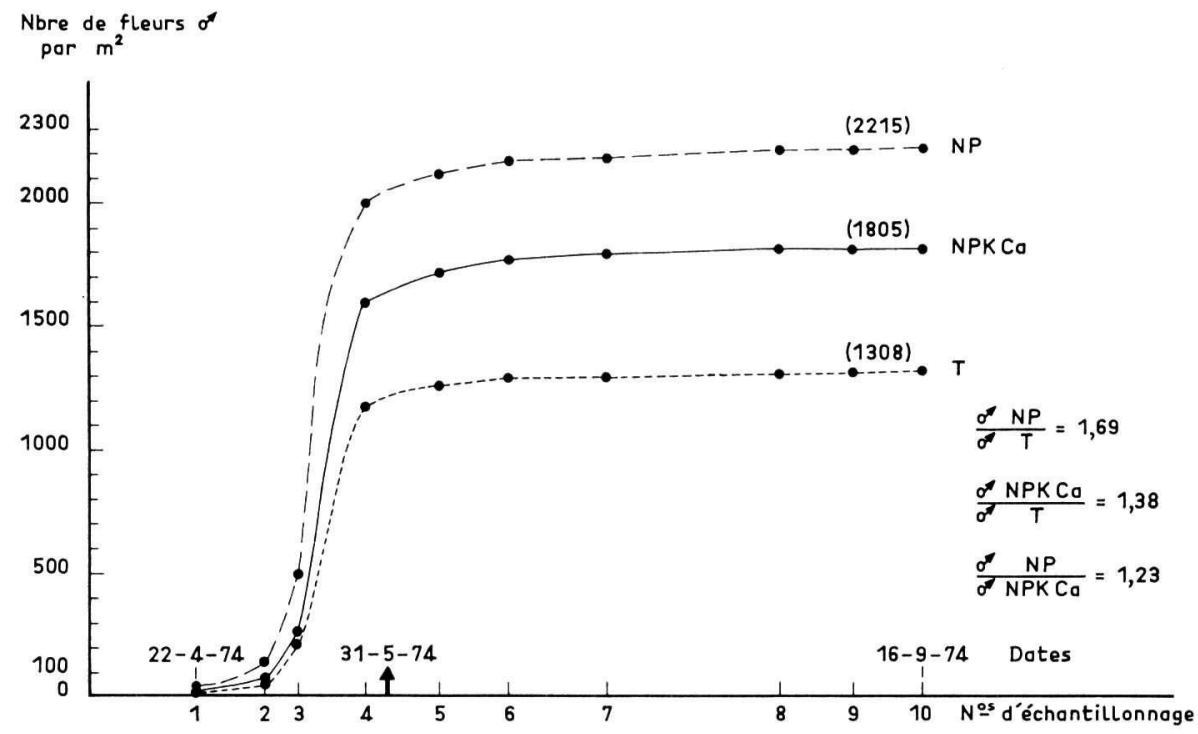

FIG. 3. - Influence de la fertilisation sur la floraison mâle (récolte de pièges) à Fougères ; courbes cumulées en fonction du temps.

The influence of fertilization on male flowering (seed traps) in Fougères; cumulation on time. 
Néanmoins, malgré ces incertitudes, les résultats de la fertilisation sont très clairs. Le traitement NP augmente le nombre de fleurs mâles de 69 p. 100. Le traitement NPKCa est dépressif par rapport à NP, mais augmente cependant la floraison mâle de 38 p. 100.

Le nombre de fleurs mâles arrivant dans les pièges a été considérable : 1308 fleurs/mètre carré dans les témoins ; 2215 fleurs/mètre carré dans les traitements NP. La chute des fleurs mâles s'est effectuée en presque totalité entre le 30 avril et le 31 mai. Une simple observation au sol laissait donc prévoir une possibilité de faînée importante dès cette date.

Par comparaison, en 1973, il est tombé dans les témoins à la même époque 58 fleurs mâles au mètre carré et dans les traitements NP 81 fleurs mâles au mètre carré. La différence n'était cependant pas significative entre ces 2 traitements.

\section{Fleurs femelles.}

Le nombre de cupules arrivant au sol pendant l'automne et l'hiver peut fournir une bonne estimation de la floraison femelle. A la fin de 1974 la plupart des cupules étaient tombées. Néanmoins quelques arbres ont gardé jusqu'au printemps 75 quelques cupules dans leurs cimes.

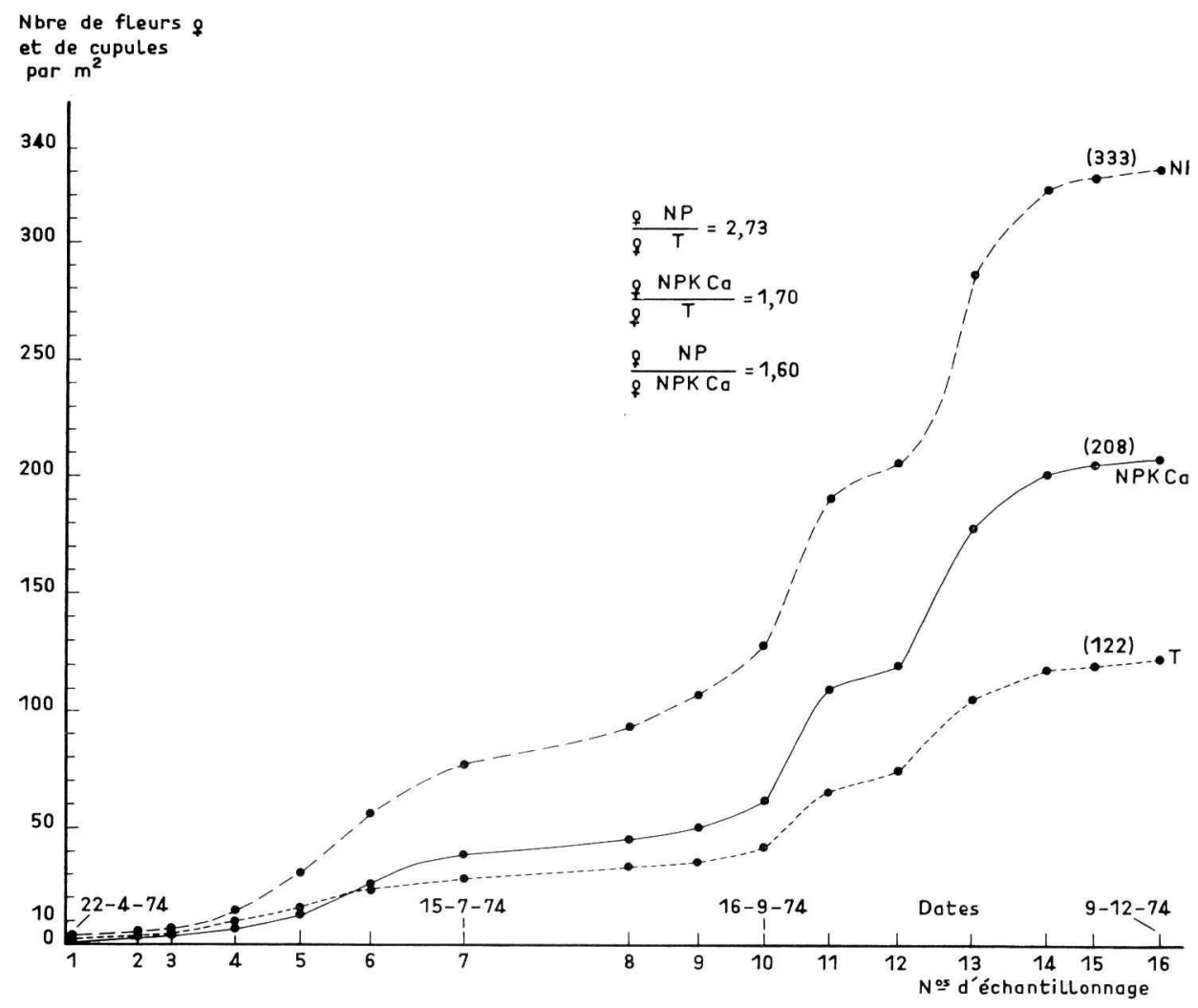

FIG. 4. - Influence de la fertilisation sur la floraison femelle (fleurs femelles et cupules à partir de pièges); courbes cumulées en fonction du temps.

The influence of fertilization on female flowering (female flowers and cupules from seed traps) ; cumulation on time. 
Pour avoir une meilleure estimation de l'induction des fleurs femelles nous pouvons y ajouter les fleurs femelles avortées au printemps (fig. 4).

L'influence de la fertilisation est très nette et visible dès le début et tout au long de la chute des fleurs femelles.

Nous obtenons ainsi (tabl. $n^{\circ}$ 6) 122 fleurs femelles tombées au mètre carré pour le témoin, 208 pour la fertilisation complète et 333 pour le traitement NP, soit un gain de 173 p. 100 de NP par rapport à T, ou un facteur multiplicatif égal à 2,73.

5.1.1.2. Influence de la fertilisation sur la production de faînes (fig. 5 et tabl. 6 ).

Nous obtenons toujours le même classement des traitements aussi bien pour le nombre de faînes totales, le nombre de faînes avortées, ou le nombre de faînes viables. Le traitement NP est de très loin le meilleur. Le traitement NPKCa est toujours dépressif par rapport à NP.

Le traitement NP permet de multiplier le nombre total de faînes par 3,21.

Le rapport faînes viables sur faînes totales est de 0,54 pour le témoin et de 0,57 pour les traitements fertilisés, mais la différence n'est pas significative (cf. tabl. 6).

On remarquera (fig. 5) que les faînes avortées ont tendance à tomber 15 jours à 1 mois plus tôt que les faînes viables, résultat classique par ailleurs.

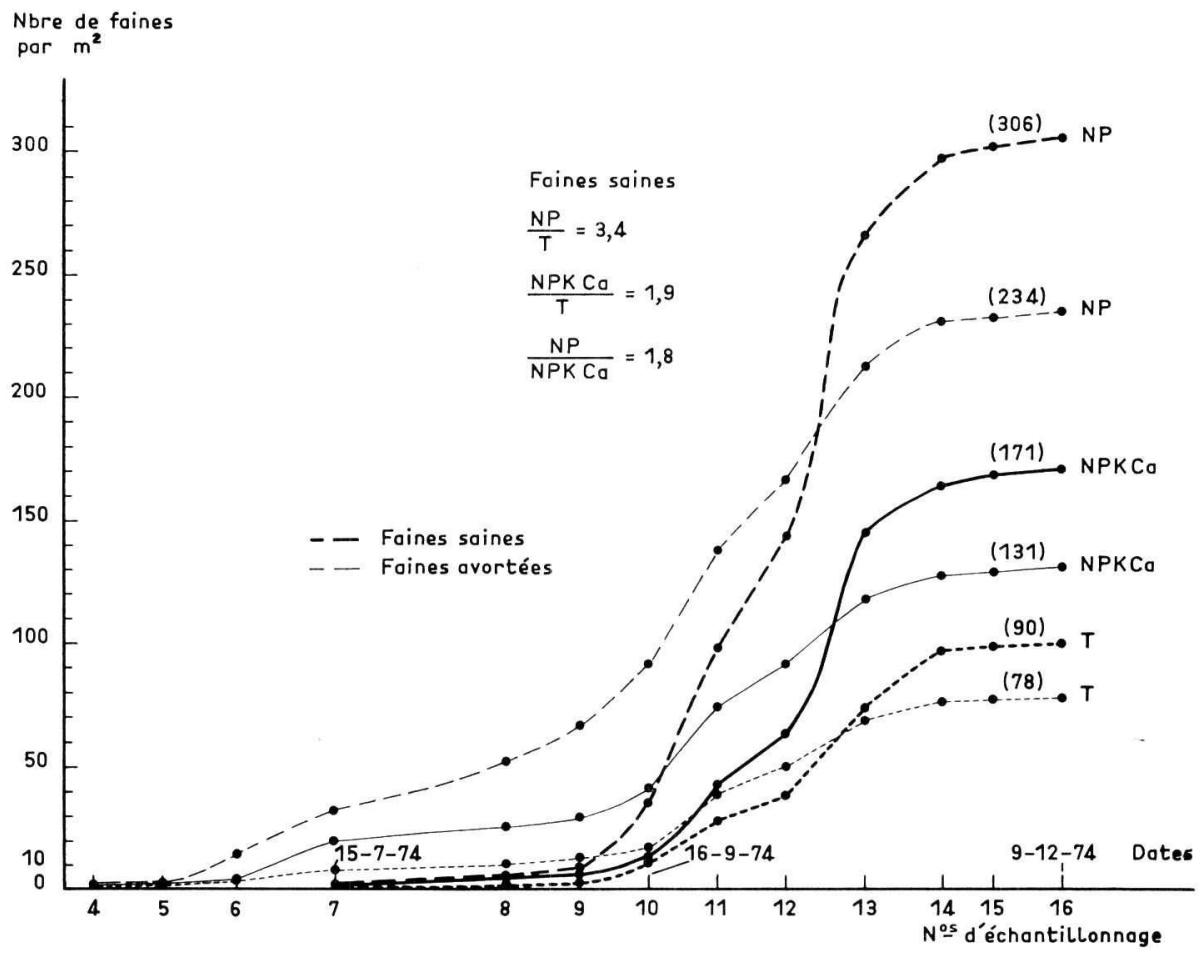

FIG. 5. - Influence de la fertilisation sur la fructification (récolte de pièges) à Fougères ; courbes cumulées en fonction du temps.

The influence of fertilization on beech nut crop (seed-traps) in Fougères ; cumulation on time, empty and sound nuts. 
TABLEAU 6

Influence de la fertilisation sur la fructification ; forêt domaniale de Fougères, pièges 1974

(Les résultats non significatifs au seuil de 5 p. 100 sont joints par un trait)

The influence of fertilization on flowering and fructification in Fougères ; collected in seed traps in 1974

\begin{tabular}{|c|c|c|c|c|c|c|c|}
\hline & $\begin{array}{c}\begin{array}{c}\text { Fleurs } \\
\text { mâles }\end{array} \\
\mathrm{m}^{2}\end{array}$ & $\begin{array}{c}\text { Fleurs femel. } \\
\text { (fleurs avort. } \\
\begin{array}{c}+ \text { cup.) } \\
\mathrm{m}^{2}\end{array}\end{array}$ & $\begin{array}{c}\begin{array}{c}\text { Faînes } \\
\text { totales }\end{array} \\
\mathrm{m}^{2}\end{array}$ & $\begin{array}{c}\text { Faînes } \\
\text { avortées } \\
\mathrm{m}^{2}\end{array}$ & $\begin{array}{c}\text { Faînes } \\
\text { viables } \\
\text { m² }^{2}\end{array}$ & $\begin{array}{l}\begin{array}{l}\text { Faînes } \\
\text { viables }\end{array} \\
\begin{array}{l}\text { faînes } \\
\text { totales }\end{array}\end{array}$ & $\begin{array}{c}\text { Poids sec } \\
\text { moyen } \\
\text { d'une faîne } \\
\text { mg }\end{array}$ \\
\hline $\mathrm{T} \ldots \ldots \ldots \ldots \ldots$ & 1308 & 122 & 168 & 78 & 90 & 0,54 & 111 \\
\hline NPKCa $\ldots \ldots \ldots \ldots$ & 1804 & 208 & 302 & 131 & 171 & 0,57 & 122 \\
\hline NP $\ldots \ldots \ldots \ldots \ldots$ & 2215 & 333 & 540 & 234 & 306 & 0,57 & 123 \\
\hline $\begin{array}{l}\text { gain NPKCa par rap- } \\
\text { port à T (en p. 100)... }\end{array}$ & 38 & 70 & 80 & 68 & 90 & & 10 \\
\hline $\begin{array}{l}\text { gain NP par rapport à } \\
T \text { (en p. 100) . . . . . }\end{array}$ & 69 & 173 & 221 & 200 & 240 & & 10 \\
\hline
\end{tabular}

Ces différents effets de la fartilisation se cumulent, et permettent une multiplication du nombre de faînes viables par 3,4 pour le traitement NP et de 1,9 pour le traitement NPKCa.

5.1.1.3. Influence de la fertilisation sur le poids moyen des faînes (tabl. 6).

La fertilisation a également un faible effet significatif sur le poids moyen d'une faîne. Le poids sec moyen d'une faîne est de 111 milligrammes pour le témoin et de 122 et 123 milligrammes pour les traitements fertilisés.

\subsubsection{Récolte au sol.}

La récolte au sol a été effectuée le 15 novembre 1974, c'est-à-dire à un moment où environ 97 p. 100 des faînes étaient déjà tombées.

Les récoltes au sol sont évidemment beaucoup moins précises que les récoltes par pièges, en raison des oiseaux et des rongeurs qui commencent à consommer des faînes dès leur chute.

II n'est donc pas étonnant de trouver par ramassage au sol un nombre beaucoup moins important de faînes que par récolte dans les pièges (fig. 6). On trouve environ 2 fois moins de faînes par cette seconde technique que par la première. dateurs.

Ceci ne veut pas dire d'ailleurs que la différence soit entièrement due aux pré-

Les opérateurs oublient obligatoirement un certain nombre de faînes par ramassage au sol. D'autre part ces récoltes au sol ont été effectuées au Nord des cimes alors que les pièges sont installés au Sud. Cette différence d'orientation, bien que nous n'ayons aucune preuve dans ce cas précis, peut avoir une influence non négligeable.

Néanmoins, si en valeur absolve les résultats des récoltes au sol sont différents 
de ceux obtenus dans les pièges, en valeur relative les résultats sont très semblables (fig. 6).

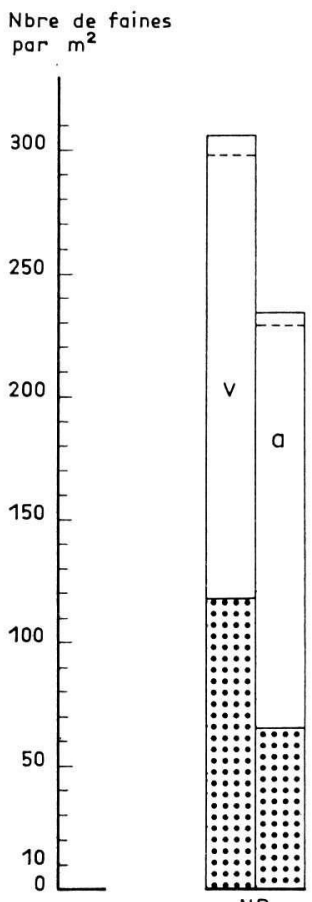

NP
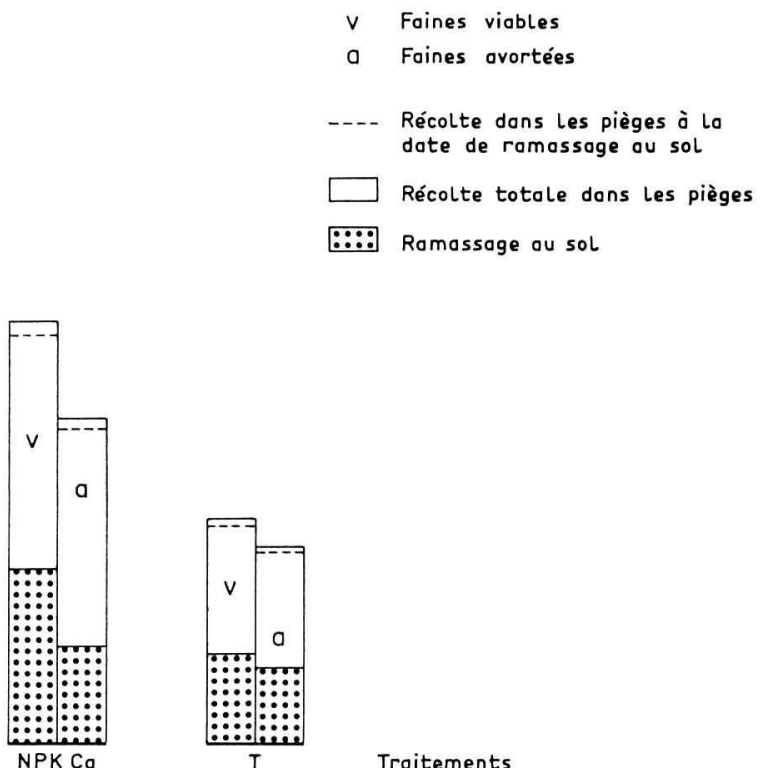

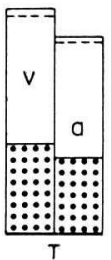

Traitements

FIG. 6. - Influence des traitements sur le nombre de faines viables et de faines avortées (pièges et ramassage au sol) à Fougères en 1974.

The influence of fertilization treatments on the number of sound and empty beechnuts collected in seed traps and on the ground in Fougères in 1974.

\section{TABLEAU 7}

Influence de la fertilisation sur la fructification; Forêt domaniale de Fougères, récolte au sol 1974

(Les résultats non significatifs au seuil de 5 p. 100 sont joints par un trait)

The influence of fertilization on fructification in Fougères ; collected on the ground in 1974

\begin{tabular}{|c|c|c|c|c|c|}
\hline & \multirow{2}{*}{$\begin{array}{l}\text { Faînes } \\
\text { totales }\end{array}$} & \multirow{2}{*}{$\begin{array}{c}\text { Faînes } \\
\text { avortées } \\
\mathrm{m}^{2}\end{array}$} & \multirow{2}{*}{$\begin{array}{c}\text { Faînes } \\
\text { viables } \\
\mathrm{m}^{2}\end{array}$} & Faînes viables & \multirow{2}{*}{$\begin{array}{l}\text { Poids sec } \\
\text { moyen d'une } \\
\text { faîne en mg }\end{array}$} \\
\hline & & & & $\overline{\text { Faînes totales }}$ & \\
\hline $\mathrm{T} \ldots \ldots \ldots \ldots \ldots \ldots \ldots$ & 65 & 30 & 35 & 0,54 & 133 \\
\hline NPKCa .. & 108 & 38 & 70 & 0,65 & 137 \\
\hline NP $\ldots \ldots \ldots \ldots \ldots \ldots \ldots$ & 183 & 65 & 118 & 0,64 & 148 \\
\hline $\begin{array}{l}\text { gain NPKCa par rapport à } T \text { (en } \\
\quad \text { p. 100) } \ldots \ldots \ldots \ldots \ldots \ldots \ldots \ldots\end{array}$ & 66 & 27 & 100 & & 3 (NS) \\
\hline $\begin{array}{l}\text { gain NP par rapport à } T \text { (en } \\
\text { p. 100) } \ldots \ldots \ldots \ldots \ldots \ldots \ldots \ldots\end{array}$ & 182 & 117 & 237 & & 11 \\
\hline
\end{tabular}


La fertilisation NP multiplie le nombre de faînes viables par 3,37 et la fertilisation complète, par 2 environ. On retrouve également l'effet de la fertilisation sur la proportion de faînes viables, mcis amplifié par rapport à la récolte dans les pièges.

\subsubsection{Effet de la fertilisation sur la nutrition minérale des semenciers.}

Des prélèvements foliaires ont été effectués le 20 août 1973 soit la première année de fertilisation (tabl. 8).

\section{TABLEAU 8}

Composition minérale des feuilles prélevées en août 1973

à Fougères (moyennes des 5 blocs)

Mineral composition of leaves in August 1973 in Fougères ; means of the 5 blocs

$\begin{array}{lllll}\text { N p. } 100 & \mathrm{P}_{2} \mathrm{O}_{5} \text { p. } 100 & \text { K p. } 100 & \text { Ca p. } 100 & \text { Mg p. } 100\end{array}$

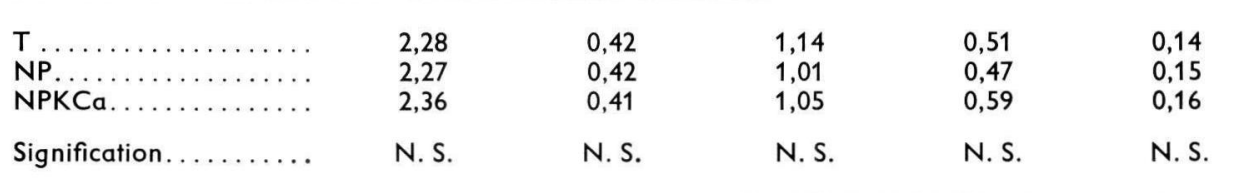

La fertilisation n'a aucun effet significatif sur la nutrition minérale des semenciers. Le niveau de nutrition du peuplement est d'ailleurs élevé en azote, moyen à élevé en phosphore, élevé en potassium et moyen en magnésium.

Seul le niveau de nutrition en calcium est faible et même beaucoup plus faible que pour les hêtraies du Nord-Est de la France. L'apport de chaux ne provoque cependant aucune augmentation significative de la teneur en calcium des feuilles.

Cet apport de chaux semble avoir un effet dépressif sur la mise à fleur et la production des faînes. La conception du dispositif ne nous permet cependant pas de savoir si cet effet dépressif par rapport à NP est dû à l'apport de chaux seul ou à l'apport conjugué de chaux et de potasse.

\section{2. - Forêt domaniale de Darney}

\subsubsection{L'influence de la fertilisation sur le nombre de cupules.}

Le phosphore seul n'a pas d'effet significatif.

Les scories ont un effet significatif (apport de $\mathrm{P}_{2} \mathrm{O}_{5}$ et $\mathrm{CaO}$ combiné) ainsi que l'apport de calcium seul.

La fertilisation complète a un effet spectaculaire et diffère significativement d'un apport de scories seules. Le meilleur traitement permet de multiplier par 3,4 le nombre de cupules (fig. 7).

\subsubsection{Influence de la fertilisation sur le nombre total des faînes (fig. 7).}

Comme précédemment, le phosphore n'a pas d'effet.

Le calcium seul et les scories ont un effet significatif à 5 p. 100. La fertilisation complète permet de multiplier le nombre de faînes par 3,6. 


\subsubsection{Influence de la fertilisation sur le nombre de faînes viables (fig. 7).}

Le phosphore et le calcium seuls n'ont pas d'effet. Les scories ont un effet significatif à 5 p. 100. La fertilisation complète permet de multiplier le nombre de faînes viables par 3,3 .

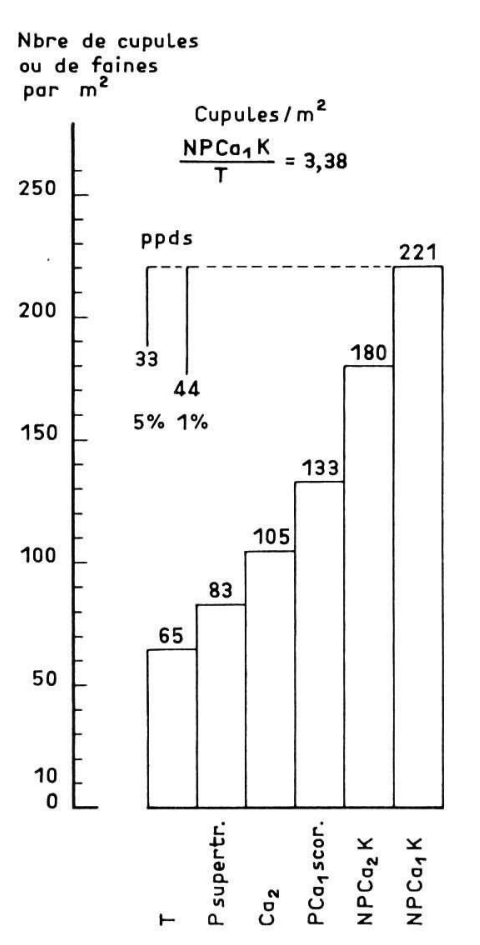

$$
\begin{aligned}
& \text { Faines totales } / \mathrm{m}^{2} \\
& \frac{\mathrm{NPCa}{ }_{1} \mathrm{~K}}{T}=3,55
\end{aligned}
$$
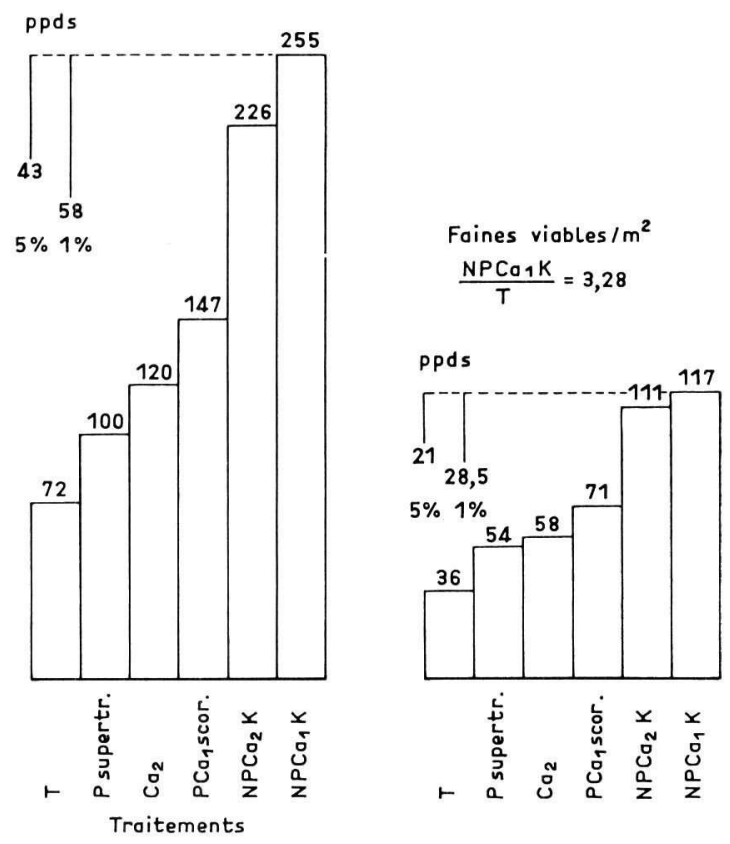

FIG. 7. - Influence de la fertilisation sur la fructification du hêtre (nombre de cupules, nombre de faines totales et nombre de faines viables) à Darney (ramassage au sol en janvier 1975).

The influence of fertilization treatments on seed crop (number of cupules, total number of beech nuts, number of sound nuts) at Darney (collected on the ground in January 1975).

\subsubsection{Interaction, effet de la fertilisation et fertilité de la station.}

Dans le modèle d'analyse de variance à 2 facteurs contrôlés (bloc et traitement) on ne met pas en évidence un effet bloc significatif au seuil de 5 p. 100. En utilisant le modèle interactif on met en évidence un effet bloc et aussi une interaction bloc $\times$ traitement significatifs. II ne semble pas cependant, que la fertilisation agisse plus dans les blocs à moder que dans les blocs à mull. La figure 2 montre les valeurs moyennes du nombre de cupules des 30 placettes unitaires. Les interactions semblent dues surtout à un comportement anormcl du bloc à mull-moder. Si après fertilisation, le nombre de cupules est plus important sur les moder et les mull-moder que sur mull, le facteur multiplicateur de la fertilisation est au moins aussi élevé sur mull que sur moder. 
5.2.5. Effet de la fertilisation sur la nutrition minérale des semenciers (tabl. 9).

L'apport d'azote améliore nettement la nutrition azotée qui est à un niveau faible à moyen dans le témoin et devient normale dans les traitements où l'azote a été épandu.

TABLEAU 9

Composition minérale des feuilles prélevées en août 1973 (moyenne des 5 blocs) ;

Forêt domaniale de Darney

Mineral composition of leaves in August 1973 at Darney; means of the 5 blocs

\begin{tabular}{llllllc}
\hline \hline & $\begin{array}{c}\mathrm{N} \\
\text { p. } 100\end{array}$ & $\begin{array}{c}\mathrm{P}_{2} \mathrm{O}_{5} \\
\text { p. } 100\end{array}$ & $\begin{array}{c}\mathrm{K} \\
\text { p. } 100\end{array}$ & $\begin{array}{c}\mathrm{Ca} \\
\text { p. } 100\end{array}$ & $\begin{array}{c}\mathrm{Mn} \\
\text { p. } 1000\end{array}$ & $\begin{array}{c}\mathrm{Mg} \\
\text { p. } 100\end{array}$ \\
\hline $\mathrm{T} \ldots \ldots \ldots \ldots \ldots \ldots \ldots \ldots$ & 1,93 & 0,29 & 1,08 & 0,48 & 2,45 & 0,10 \\
$\mathrm{P} \ldots \ldots \ldots \ldots \ldots \ldots \ldots \ldots$ & 1,93 & $0,36 *$ & 1,07 & $0,59 *$ & 3,22 & 0,10 \\
$\mathrm{Ca}_{2} \ldots \ldots \ldots \ldots \ldots \ldots \ldots$ & 1,94 & 0,28 & $0,87 *$ & $0,74 *$ & 3,72 & 0,12 \\
$\mathrm{PCa}_{1} \ldots \ldots \ldots \ldots \ldots \ldots \ldots$ & 1,92 & $0,35 *$ & 1,05 & $0,64 *$ & 3,56 & 0,12 \\
$\mathrm{NPCa} \mathrm{a}_{1} \mathrm{~K} \ldots \ldots \ldots \ldots \ldots \ldots$ & $2,13 * *$ & $0,33 *$ & 1,07 & $0,59 *$ & 3,30 & 0,10 \\
$\mathrm{NPCa} \mathrm{a}_{2} \mathrm{~K} \ldots \ldots \ldots \ldots \ldots$ & $2,20 * *$ & $0,31 *$ & $0,98 *$ & $0,64 *$ & 3,73 & 0,12 \\
\hline
\end{tabular}

L'apport de phosphore qu'il soit sous forme de supertriple ou de scories améliore significativement la nutrition en cet élément. L.'apport de potassium n'a aucun effet.

Néanmoins, les doses élevées de calcium (1 500 kılogramme de CaO à l'he .tare) diminuent nettement le niveau du potassium dans les feuilles.

Enfin, la teneur en calcium des feuilles augmente dans les traitements où il y a eu apport de calcium aussi bien par les scories que par le calcaire.

\section{6. - Discussion et conclusions}

L'essai de Fougères montre que sur un sol à mor-moder développé sur limons, fréquemment représenté dans les hêtraies de l'ouest de la France, l'apport de 200 kilogrammes d'azote et de 150 kilogrammes de phosphore à un peuplement de hêtre âgé de 120 à 140 ans a un effet considérable sur la fructification.

C'est ainsi que la fertilisation agit tout d'abord sur la mise à fleur en augmentant le nombre de fleurs mâles de 69 p. 100 et surtout en multipliant le nombre de fleurs femelles par 2,7.

La fertilisation agit enfin en augmentant le nombre de faînes viables par rapport au nombre de faînes avortées.

Ces différents effets se conjuguent et permettent de multiplier le nombre de faînes viables par 3,4.

C'est ainsi qu'une fertilisation azotée et phosphatée permet de passer de 90 faînes viables à 306 faînes viables au mètre carré (récolte dans des pièges), ou de 35 faînes viables à 118 faînes viables par mètre carré (récolte au sol). 
Les résultats obtenus en forêt domaniale de Darney confirment parfaitement ceux obtenus dans l'Ouest de la France. Le facteur multiplicateur de la faînée par fertilisation est du même ordre de grandeur : 3,3 environ.

Les apports de phosphore seul n'ont aucun effet significatif. L'apport de calcium seul à raison de 1500 kilogrammes/hectare de $\mathrm{CaO}$, a un effet significatif à 5 p. 100 sur le nombre de cupules, c'est-à-dire sur les potentialités de fructification ainsi que sur le nombre total de faînes. II n'a par contre pas d'effet sur le nombre de faînes viables.

L'apport de scories (240 kilogrammes de $\mathrm{P}_{2} \mathrm{O}_{5}+675$ kilogrammes de $\mathrm{CaO} / \mathrm{ha}$ ) a un effet positif sur le nombre de cupules, le nombre total de faînes et le nombre de faînes viables.

Une fertilisation complète a un effet considérable sur tous les paramètres de la fructification. Cet effet semble surtout attribuable à l'apport d'azote; le potassium ne semble jouer aucun rôle.

On remarquera dans les traitements complets qu'un apport de calcium supplémentaire (1500 kilogrammes de $\mathrm{CaO}$ au lieu de 675 kilogrammes de $\mathrm{CaO}$ à l'hectare) a un faible effet dépressif significatif sur les potentialités de fructification (nombre de cupules).

Ces résultats sont à rapprocher de ceux de Fougères qui mettent en évidence un effet dépressif de la fertilisation NPKCa par rapport à une fertilisation NP.

Une fertilisation de type NP semble donc bien la meilleure. L'apport de phosphore sous forme de scories semble souhaitable.

Le résultat le plus intéressant de l'essai de Darney est peut-être de montrer que la fertilisation a un effet sur la fructification même sur des peuplements de productivité élevée et cela 2 à 3 ans après l'apport des engrais.

Cet effet semble assez indépendant du type de station, puisqu'il est aussi important sur mor (Fougères) que sur moder et mull acide (Darney).

Les premiers résultats de ces 2 essais nous permettent déjà de conseiller une fertilisation de ce type dans des peuplements situés dans des conditions écologiques voisines, c'est-à-dire sur sols à moder ou à mor développés sur limon ou sur sols à moder ou à mull acide développés sur grès.

La plupart des grandes hêtraies de Normandie et des Basses-Vosges se trouvent dans ces conditions écologiques. La fertilisation de semenciers peut trouver une application immédiate dans les peuplements classés pour la production de graines. Pour la régénération naturelle il reste à étudier un éventuel effet dépressif de la fertilisation sur la croissance des semis par une concurrence accrue de la strate herbacée.

Plusieurs autres questions restent cependant à résoudre. On peut se demander si un apport d'azoie seul ne serait pas suffisant et il importe également de savoir pendant combien d'années les engrais seront efficaces, puisque les faînées abondantes peuvent être espacées de plusieurs années (3 à 10 ans). A Darney l'effet de la fertilisation est toujours très important 3 ans après l'apport principal et 2 ans après le dernier apport d'azote. La faînée 1976, qui s'annonce très bonne permettra de poursuivre les observations aussi bien à Fougères qu'à Darney.

Enfin il serait nécessaire de multiplier les essais de façon à couvrir toute la variabilité des hêtraies économiquement intéressantes et en particulier les hêtraies des plateaux calcaires du Nord-Est de la France.

Reçu pour publication en décembre 1976. 


\section{Remerciements}

Nous remercions M. Mallard, Chef de Centre de Rennes et MM. Mormiche et Guinard successivement Chefs de Centre d'Epinal Nord ainsi que le personnel de ces deux centres de l'Office National des Forêts, qui nous ont donné toutes les facilités pour l'installation des dispositifs expérimentaux.

Nous remercions MM. Charlon et Bonischot de la Société Nationale des Scories Thomas qui ont participé à l'élaboration et au financement du dispositif expérimental de Darney.

Nous remercions MM. Badia et Masson de la Station de Biométrie du C.N.R.F. qui nous ont conseillé et aidé pour le traitement statistique des données.

Enfin les ramassages au sol, longs et fastidieux, ont pu être réalisés grâce au dévouement de la quasi-totalité du personnel de la station de sylviculture et de la station de recherches sur les sols forestiers et la fertilisation.

\section{Summary}

\section{The influence of fertilization on the fructification of beach (Fagus silvatica L.)}

A fertilization experiment in the West of France (Fougères) and another one in the East of France (Darney) showed a considerable effect of fertilization on the fructification of beech.

In Fougères the number of male flowers was 70 p. 100 greater in fertilized plots, than in non fertilized plots, the female flowering was increased 2.7 times by fertilization. The percentage of sound beech nuts is also higher after fertilization.

Those different effects increase jointly the number of sound beech nuts for about 3.4 times in Fougères and 3.38 times in Darney.

The effect of fertilization seems to be due to the application of nitrogen and to a certain extend also of phosphor. A NP fertilization can therefore be recommended.

Increased fructification was obtained even in stands of high yield (Darney) and also in older stands (140 years, Fougères). The fertilization effect seems to be independant of the types of humus: fructification is increased as well on sites with raw humus (mor), moder and acid mull. In Darney the effect was obtained even three years after the application of the fertilizers.

Some questions have still to be answered :

- the duration of the effect of an unique application of a fertilizer,

- the effect on sites with mesotrophic, autrophic mull and calcareous mull.

\section{Zusammenfassung}

\section{Der Einfluss der Düngung auf die Fruktifikation der Buche (Fagus silvatica L.)}

An einem Düngungsversuch in Westfrankreich (Fougères) und einem Düngungsversuch in Ostfrankreich (Darney) konnte gezeigt werden, dass die Düngung (NP) einen bedeutenden Einfluss auf die Fruktifikation der Buche hat.

In Fougères wurde die Anzahl der männlichen Blüten un 70 p. 100 erhöht, während die der weiblichen Blüten das 2.7 fache betrug. Durch die Düngung wurde auch der Anteil an gesunden Bucheckern erhöht. Diese werschiedenen Düngerwirkungen führten dazu, dass die Anzahl der gesunden Bucheckern um das 3.4 fache (Fougères) bzw. 3.38 fache (Darney) zunimmt.

Die Wirkung der Düngung scheint vor allem auf den Stickstoff und bis zu einem gewissen Grad auch auf den Phosphor zurückzuführen sein und eine NP Düngung kann daher empfohlen werden.

Der Düngungseffekt zeigł sich sowohl in Beständen hoher Wuchsleistung (Darney) als auch in älteren Beständen (140 jährig, Fougères) ; er scheint bis zu einem gewissen Grad unabhängig vom Humustyp, da sowohl auf Rohhumus und Moder, als auch auf sauren Mull annähernd gleiche Ergebnisse erzielt wurden.

In Darney zeigle sich, dass die Düngung noch drei Jahre nach der Ausbringung wirkt.

Mehrere Fragen stehen jedoch noch offen :

- Wirkungsdauer einer einmaligen Düngung,

- Düngungseffekt auf mesotrophen und eutrophen Mullstandorten bzw. auf Kakkmullstandorten. 


\section{Références bibliographiques}

BIENAIME E., GIGOUT M., 1975. Bibliothèque statistique, fichiers standards. Station de Biométrie du C.N.R.F.-I.N.R.A. Document ronéotypé, no 75/12, 16 p.

BORCHERS K., GUSSONE H.-A., KRAMER H., 1964. Ergebnisse von Stickstoffdüngungsversuchen in den niedersächsischen Forstämtern Boffzen, Neuhaus und Schöningen. Aus dem Walde, no 8, pp. 75-108.

DECOURT N., 1973. Tables de production pour les forêts françaises. E.N.G.R.E.F., Centre de Nancy, $49 \mathrm{p}$.

GARBAYE J., LEROY Ph., 1974. Influence de la fertilisation sur la production de glands en forêt de Bercé et de Boulogne. Rev. For. Franç., no 3/1974, pp. 223-228.

HAMILTON G. J., CHRISTIE J. M., 1971. Forest Management Tables (metric). Forestry Commission Booklet, no 34, 201 p.

HAUSSER K., 1971. Düngungsversuche zu 70-90 jährigen Buchenbeständen auf der Schwäbischen Alb. AFJZ, 1971, 8/9, pp. 225-233.

LE GOFF N., 1974. La croissance du hêtre en France : uitilisation possible des tables de production étrangères pour suivre l'évolution des peuplements. I.N.R.A.-C.N.R.F., Station de Sylviculture et de Production, document interne, no 74/3, 73 p.

LE TACON F., OSWALD H., 1975. Premiers résultats d'un essai de fertilisation minérale sur la fructification du hêtre en forêt domaniale de Fougères (Ille-et-Vilaine). Document ronéotypé, C.N.R.F. Nancy, 15 p.

LE TACON F., OSWALD H., 1976. Influence de la fertilisation minérale sur la fructification du hêtre (Fagus silvatica) en forêt domaniale de Darney (Vosges). I.N.R.A.-C.N.R.F. Document ronéotypé, 9 p.

MASSON J. P., BADIA J., GIGOUT M., 1975. Bibliothèque de programmes d'analyse de variance. Station de Biométrie du C.N.R.F.-I.N.R.A. Document ronéotypé, no 75/11, 10 p.

SCHOBER R., 1972. Die Rotbuche. J. D. Sauerländers Verlag, Frankfurt a. M., 333 p.

TOUTAIN F., 1966. Etude du sol et des eaux de la forêt de Fougères (Ille-et-Vilaine). Thèse Faculté des Sciences de Paris.

WEISSEN. 1970. Résultats de l'essai de fertilisation de Bertrix (Belgique). Communication personnelle 